\title{
NanoMed 2020 - Enabling the European nanomedicine area until 2020
}

Olivier Fontaine (ETPN), Patrick Boisseau (ETPN Chairman and Nanomedicine Business developer at CEA-LETI) and Dr. Klaus-M. Weltring (Managing Director Bioanalytikmuenster e. V.).

Nanomedicine, specifically the use of nanotechnologies for medical applications, has been around for the last 30 years, although it was not designed as such in the very beginning. It is not a futuristic vision anymore, and products are already entering the market for the benefit of the patients and society: today, 44 nano-delivery products have received market approval in the USA (including 18 pharmaceutical products) as well as 15 products in the fields of imaging and diagnostics. Many more are currently under clinical trials (more than 70 worldwide) and clinical uptake of key products can already be witnessed in the field of in vivo imaging for surgical procedures for example.

As more products and case studies are emerging, a global landscape is gradually appearing, highlighting crucial issues, gaps and needs that need to be addressed urgently for nanomedicine to keep its pace of development. Outstanding research is currently being performed throughout Europe, but with limited market outputs. For the last few years, the concept of "translational nanomedicine" has been at the core of many concerns and issues explaining this lack of commercial uptake have been identified. Among these issues, the lack of industrial awareness and the poor industrial feasibility of European research are commonly cited reasons.

This is especially relevant in the field of therapeutics where uptake by large pharmaceutical industries is needed to cover the costs of clinical trials. For other fields such as in-vitro diagnostics, companies could manage on their own but there are very few start-ups on the market due to a highly uncertain, complex and changing regulatory landscape, with difficulties to earn sufficient funds and to find the proper guiding support for proof of concept, a crucial step that propels the company if managed efficiently.

At the end of 2013, recommendations will be made by the European Commission to structure the orientation and priorities of the next European framework program called Horizon 2020, which will fund research projects at the European level from 2014 until 2020. It has already been established that Excellence Science, Industrial leadership and Societal Challenges will be the three pillars of the next European framework program. To support them, six Key Enabling Technologies (KETs) have been identified with strategic importance: nanotechnologies, advanced materials, micro- and nano-electronics, photonics, biotechnology, and advanced manufacturing.

In this context, nanomedicine has more than one role to play: as a highly interdisciplinary field, this technology has the potential and necessity to combine most of the KETs to deliver advanced products and to keep proving to be an essential enabling instrument for personalized, targeted and regenerative medicine. Beyond that, nanomedicine will offer tremendous tools to cope with societal health challenges such as an aging population and disparate access to treatments: by enabling improved and cost-effective health-care, it will embody one crucial factor for making medicines and treatments more available and affordable.

Bearing in mind that according to BCC Research (Market report, 2012) the global nanomedicine market is expected to grow from $\$ 63.8$ billion in 2010 to $\$ 130.9$ billion by 2016, it can be ensured that economic and industrial return on investment will be considerable, if research is carefully framed and pushed forward.

Recently, an unprecedented effort of consolidating the area of Nanomedicine research has been undertaken in order to deliver valuable inputs for and to promote nanomedicine in the Horizon 2020 framework program, giving rise to NanoMed 2020, a project funded with a contribution by the European Commission under the Work Program FP7-HEALTH-2012. This 18 months European Coordinated Action started in September 2012 and will end in February 2014 with key deliverables and recommendations for the European Commission for funding nanomedicine related research activities. Making Europe a center for profitable nanomedicine research, development and translation is the goal of this project.

The objectives are ambitious yet necessary and will need the joined forces of all actors and involved partners; namely the European Technology Platform on Nanomedicine ETPN (operated by VDI/VDE Innovation + Technik GmbH), Germany, the European Foundation for Clinical Nanomedicine (CLINAM Foundation), Switzerland, the National Institute of Health Carlos III (ISCIII), Spain, Bioanalytik-muenster e.V (BioMS), Germany, Nanobiotix SA, France, the Fondazione Don Carlo Gnocchi ONLUS (FDG), Italy, and SINTEF Materials and Chemistry, Norway.

In the process of drafting concepts for more efficient funding programs and translation strategies, NanoMed 2020 plans to lead various and complementary workshops, ranging from the federation of the nanomedicine community and the introduction of novel communication tools to the implemen- 
tation of expert workshops on societal challenges and the settings for long-term international cooperation.

Special attention is given to the fact that academic research groups need to better understand the market and the medical needs. Furthermore, there are a large number of pharmaceutical and med-tech companies that have a comprehensive and direct access to the market but these companies struggle to handle radical innovation. At the same time, there is a small number of SMEs trying to introduce new innovation onto the market, but these few companies are too small to exploit the large potential of nanomedicine. A consolidated pan-European approach to interface these stakeholders is therefore very much needed to bring nanomedicine products more efficiently to patients.

With such considerations in mind, a "Translation Hub" has been at the heart of many recommendations and will embody one of the two specific activities nanomedicine will propose under NanoMed 2020. Such a mediator structure will serve as a liaising agent and supporter for early-stage projects until they reach clinical trials where transfer to large companies would be possible for further development. The translation hub will also accompany projects for non-clinical applications like in-vitro diagnostics. Education of academia to industrial development constraints, evaluation of projects with the industrial perspective and feasibility, and advices from translational experts to accompany the most promising research are additional services that will be provided in order to initiate a sustainable open innovation model for nanomedicine in Europe.

Inspired by the US Nano-Characterization Lab (NCL), NanoMed 2020 members have outlined the crucial importance of a similar infrastructure in Europe to boost its sovereignty in the field.

In close collaboration with the Translation Hub, a group such as the European Nano-Characterization Laboratory would provide a comprehensive set of characterization parameters (physical, chemical, in vitro and in vivo biological properties) allowing researchers to speed up the approval process for their particles developed to solve problems that affect

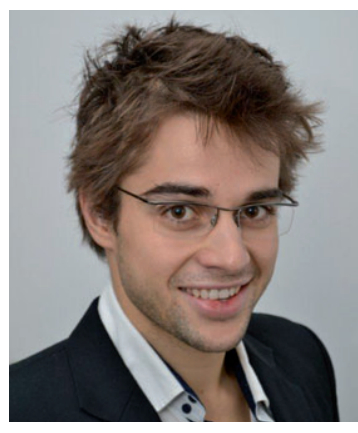

Olivier Fontaine is trained as a multidisciplinary engineer at Ecole Centrale de Lille (France) and finalized in September 2012 a double degree program at Cranfield University (UK) with a Nanomedicine MSc. His MSc thesis on the nanomedicine commercial landscape, co-supervised by Dr Bojan Boskovic from Cambridge Nanomaterials Technology Ltd, allowed him to gain advanced knowledge, skills and patients' health. A direct link with the European Medicines Agency (EMA) is required to facilitate the approval of nanomedicine products based on the characterization report delivered by the European Nano-Characterization Laboratory. It will contribute to the European competitiveness of nanomedicine products and tools by increasing the industry readiness of research offerings and thus stimulate privatesector investment in this area.

It is still under discussion whether this infrastructure would rely or not, only on existing laboratories and clusters of excellence in Europe, setting up the priority on networking and coordinating the existing centers.

The nanomedicine partners will have to team up with other stakeholders and groups (IMI, EATRIS ...) under Horizon 2020 and for joint activities with pharma- (EPFIA) and diagnostic(COCIR) companies on common issues such as the needs for education on translation and increased public awareness.

On a different front, NanoMed 2020 members are currently bringing together all resources available and involved players to unite the community in delivering the most exhaustive mapping of nanomedicine activities ever established across Europe. In addition to being an outstanding visual tool showing the impact and importance of nanomedicine in current European activities, this database will serve as a stepping stone in highlighting the economic and industrial importance of nanomedicine in Europe.

An update of the ETPN Strategic Research and Innovation Agenda encompassing the latest trends and applications will also be a key milestone of the project, keeping its fundamental role of advising national policies and the European Commission on coming research funding priorities. Reflecting well the policy of its contributors, this strategic document will explicitly include for the first time an Innovation dimension in its roadmap, encouraging actions to be taken in this regard.

More than delivering nanomedicine inputs for the future European research priorities until 2020, NanoMed 2020 will drive the long-term development and capacities of this field.

practical experience in the identification and understanding of drivers, needs and players in the supply chain of nanomedicine.

Building upon his expertise, Olivier Fontaine is now working at the secretariat of the European Technology Platform on Nanomedicine (ETPN, Germany) as an International Volunteer from Nanobiotix (France). Taking an active role in the NanoMed 2020 European project and in the elaboration of strategic documents, he is currently in charge of mapping the nanomedicine actors and initiatives at the European level, along with contributing to novel communication tools and establishing the state-of-the art activities and needs in translational nanomedicine. 


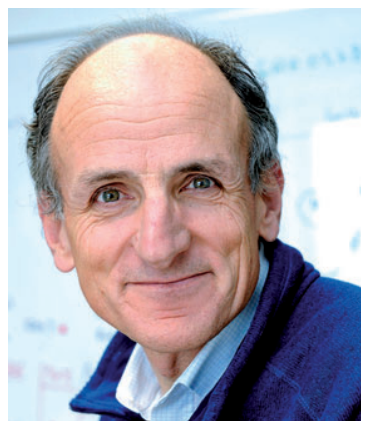

Patrick Boisseau joined the French Atomic Energy and Alternative Energies Commission (CEA) in 1987 to work for 7 years as academic research fellow in plant biology. He then spent 4 years at the Foresight \& Strategy Division at the CEA headquarters as expert on strategy in life sciences and environment. From 2001 to 2004, he was committed to the design, organization and funding of the NanoBio innovation cluster in Grenoble. The NanoBio center is an integral part of the Minatec Innovation Center, the model for France's competitive clusters and \#1 European center for micro- and nanotechnologies. From 2004 until 2008, he was coordinator of the European network of excellence in nanobiotechnology, Nano2Life (www.nano2life.org). This network of excellence integrates 23 full academic partners and 41 associate companies $(>400$ scientists) in a comprehensive joint program of activity. Since then, he has been part of more than 12 European projects and main coordinator of 5 Framework Projects. Since 2012, he has been elected Chairman of the European Technology Platforms on Nanomedicine that he joined in 2005. Since 2008, he has been Programme Manager on nanomedicine, at CEA-Leti.; 15 scientists are devoted there to the preclinical and clinical development of Lipidots $®$ lipid nanocarriers. Since 2012 he is head of the Strategic Planning on Healthcare at CEATech. Patrick Boisseau is graduate of the Institut National Agronomique (1983) and of the Ecole Nationale du Génie Rural, des Eaux et des Forêts (1985). He holds a Master's Degree in Human Nutrition (2005).

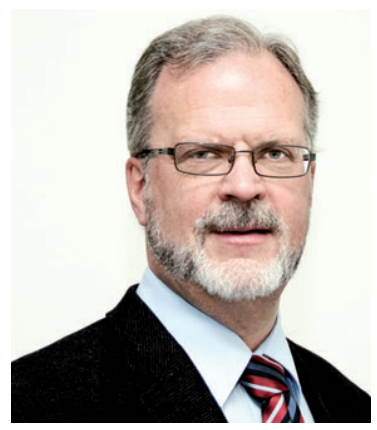

Dr. Klaus-Michael Weltring is a molecular biologist by training with a $\mathrm{PhD}$ and a Habilitation degree from the University of Münster. Since 2001 he is the managing director of bioanalytik-muenster responsible for the development of the Münster region into a leading nanobioanalytic location at the European level. He has set up a local network of researchers from different disciplines and SMEs and organizes the marketing of the region at international events and fairs. Currently he manages a network of 11 local companies funded by the national ZIM NEMO program, which has the goal to develop SOP-based methods for the characterization of nanomaterials in biological systems.

Between 2003 and 2008 he was the deputy-coordinator of the Nano2Life Network of Excellence and leader of the "ELSA" Board in this network. In this project he organized two expert workshops on ELSA aspects of nanobiotechnology. Until recently he co-managed the Nanomedicine Round Table and the EuroNanoBio project (FP7 CSA projects). In addition he is a member of the Executive Board of the ETP Nanomedicine leading the ELSA Advisory Group of this platform. In Summer 2010 he co-organized the event "Nanomedicine in Europe: present and for the future" at the European Parliament. Currently he is a partner in the successful EU proposal NanoMed 2020, which will perform a study how to develop nanomedicine in Europe. 